NBER WORKING PAPER SERIES

\title{
EFFICIENT KIDNEY EXCHANGE: COINCIDENCE OF WANTS IN A STRUCTURED MARKET
}

\author{
Alvin E. Roth \\ Tayfun Sönmez \\ M. Utku Ünver \\ Working Paper 11402 \\ http://www.nber.org/papers/w11402 \\ NATIONAL BUREAU OF ECONOMIC RESEARCH \\ 1050 Massachusetts Avenue \\ Cambridge, MA 02138 \\ June 2005
}

We thank Audrey Bohnengel, Kevin Cheung, Francis Delmonico, Michael Goemans, Selçuk Karabati, Jonathan Kopke, Michael Rees, Susan Saidman, JunWako and SteveWoodle for discussions and comments, Rui Dong for her research assistance, Hüseyin Çankaya for his assistance on drawing the figures. Sönmez acknowledges the research support of KoçBank via the KoçBank scholar program and Turkish Academy of Sciences in the framework of the Young Scientist Award Program via grant TS/TÜBAGEBúIP/2002-1-19, and Roth and Ünver acknowledge support from the NSF via grant no: 0338619. Any errors are our own. The views expressed herein are those of the author(s) and do not necessarily reflect the views of the National Bureau of Economic Research.

(C2005 by Alvin E. Roth, Tayfun Sönmez, and M. Utku Ünver. All rights reserved. Short sections of text, not to exceed two paragraphs, may be quoted without explicit permission provided that full credit, including (c) notice, is given to the source. 
Efficient Kidney Exchange: Coincidence of Wants in a Structured Market

Alvin E. Roth, Tayfun Sönmez, and M. Utku Ünver

NBER Working Paper No. 11402

June 2005

JEL No. C7, C6

\begin{abstract}
Patients needing kidney transplants may have willing donors who cannot donate to them because of blood or tissue incompatibility. Incompatible patient-donor pairs can exchange donor kidneys with other such pairs. The situation facing such pairs resembles models of the "double coincidence of wants," and relatively few exchanges have been consummated by decentralized means. As the population of available patient-donor pairs grows, the frequency with which exchanges can be arranged will depend in part on how exchanges are organized. We study the potential frequency of exchanges as a function of the number of patient-donor pairs, and the size of the largest feasible exchange. Developing infrastructure to identify and perform 3-way as well as 2-way exchanges will have a substantial effect on the number of transplants, and will help the most vulnerable patients. Larger than 3-way exchanges have much smaller impact. Larger populations of patient-donor pairs increase the percentage of patients of all kinds who can find exchanges.
\end{abstract}

\author{
Alvin E. Roth \\ Harvard University \\ Department of Economics \\ Littauer 308 \\ Cambridge, MA 02138-3001 \\ and NBER \\ al_roth@harvard.edu

\section{Tayfun Sönmez} \\ Koç University \\ Department of Economics \\ Rumelifeneri Yolu, Sariyer, 34450 \\ Istanbul Turkey \\ tsonmez@ku.edu.tr
}

M. Utku Ünver

Koç University

Department of Economics

Rumelifeneri Yolu, Sariyer, 34450

Istanbul Turkey

uunver@ku.edu.tr 


\section{Introduction}

In 2003 there were 8,665 transplants of deceased donor kidneys for the approximately 60,000 patients waiting for such transplants in the U.S.. While waiting, 3,436 patients died. There were also 6,464 kidney transplants from living donors. ${ }^{1}$

A patient is often unable to receive a willing live-donor's kidney, because of blood-type incompatibility or antibodies to one of the donor's proteins ("positive crossmatch"). Recently a few "paired kidney donations" have been performed between two such incompatible patientdonor pairs: the donor in each pair gives a kidney to the other pair's compatible patient. The National Organ Transplant Act of 1984 makes it illegal to buy or sell a kidney, and so incompatible patient donor pairs are faced squarely with Jevons' classic problem of the "double coincidence of wants:"

Jevons (1876), Chapter 1: "The first difficulty in barter is to find two persons whose disposable possessions mutually suit each other's wants. There may be many people wanting, and many possessing those things wanted; but to allow of an act of barter, there must be a double coincidence, which will rarely happen. ... the owner of a house may find it unsuitable, and may have his eye upon another house exactly fitted to his needs. But even if the owner of this second house wishes to part with it at all, it is exceedingly unlikely that he will exactly reciprocate the feelings of the first owner, and wish to barter houses. Sellers and purchasers can only be made to fit by the use of some commodity... which all are willing to receive for a time, so that what is obtained by sale in one case, may be used in purchase in another. This common commodity is called a medium, of exchange, because it forms a third or intermediate term in all acts of commerce."

Partly because of the difficulty of finding these double coincidences, there have been few such exchanges: In the fourteen transplant centers in New England (The United Network for Organ Sharing - UNOS - region 1), five such 2-way exchanges had been conducted as of December 2004 (cf. Delmonico, 2004). There have also been a very few 3-way exchanges (Lucan et al. $2003)^{2}$, two in the United States, by Dr. Robert Montgomery's program at Johns Hopkins. All surgeries in an exchange are done simultaneously (so 2-way exchange requires four simultaneous surgeries, and 3-way requires six), and so larger exchanges pose more logistical difficulties.

\footnotetext{
${ }^{1}$ The U.S. Scientific Registry of Transplant Recipients http://www.ustransplant.org/srtr.php. Live donation of kidneys is possible because people have two kidneys, and because healthy people suffer little risk from donating one.

${ }^{2}$ In a three-way exchange, the donor from one pair gives a kidney to the patient of a second pair, whose donor gives to the patient in a third pair, whose donor gives to the patient in the first pair.
} 
One theme of the present paper is that some of the difficulties that Jevons attributes to the absence of a medium of exchange will also loom large whenever a thick market is lacking. Even with a medium of exchange, Jevons' second house owner would be reluctant to part with his house if no suitable third house could be found. And we will show that, even without a medium of exchange, the problem of the coincidence of wants can be substantially ameliorated by the presence of an appropriate market structure.

One reason there have been so few exchanges is that until recently there have been no databases of incompatible patient-donor pairs: incompatible donors were simply not further considered. This is starting to change. In September, 2004, the New England Program for Kidney Exchange, proposed by Drs. Francis Delmonico, Susan Saidman, and the three authors of this paper was approved by the Renal Transplant Oversight Committee of New England. (It will be administered through the New England Organ Bank.) Databases for identifying kidney exchanges have also been initiated in Ohio and Baltimore. As these centers start to open their doors to patient-donor pairs, one of the primary tasks is to design a clearinghouse that can identify efficient sets of feasible exchanges among incompatible patient-donor pairs (cf. Roth, Sönmez, and Ünver, 2004a,b, 2005, Segev et al. 2005).

The "coincidence of wants" for kidney exchange has a structure determined in part by the blood types of the patients and donors. Consequently it is possible to bring simple theory to bear on the question of how efficient such exchange can be, operating just as a gift-exchange, in the absence of any medium of exchange. ${ }^{3}$ Computational results on real and simulated patient data (Saidman et al. 2005) suggest that as the available population of incompatible patientdonor pairs grows, an increasing percentage of patients will be able to receive a transplant via a two way exchange, that three-way exchanges will continue to be important for achieving efficient exchange, and that most of the efficiency gains from exchanges larger than 2-way are captured by including 3-way exchanges. ${ }^{4}$ In the present paper we explore why this is the case. That is, we investigate the structure of efficient exchange, without a medium of exchange, when supply and demand are mediated by blood types.

Furthermore, we will prove that, under the conditions of supply and demand that can normally be expected in a population of incompatible patient-donor pairs available for exchange, if we abstract away from tissue type incompatibilities and look at only the barriers to exchange

\footnotetext{
${ }^{3}$ There is also a literature devoted to the discussion of whether the ban on the buying and selling of kidneys should be repealed. See e.g. Becker and Elias (2002), and the survey of this literature in Nadel and Nadel (2005).

${ }^{4}$ We have recently been able to confirm this on a database of patient-donor pairs assembled in Ohio by Dr. Steve Woodle, Dr. Michael Rees, Jonathan Kopke and their colleagues in the Paired Donation Kidney Consortium.
} 
caused by blood-type incompatibilities, all efficient exchanges can be accomplished in exchanges involving no more than four incompatible pairs.

\section{Background}

There are four blood types A, B, AB, and $\mathrm{O}$ (corresponding to the presence of proteins $\mathrm{A}$ or $\mathrm{B}$, or both together, or neither) and blood-type incompatibility has a very well-defined structure: a patient may not receive the kidney of a donor whose blood contains one of the A,B proteins that the patient does not have. Thus patients with blood type $\mathrm{O}$ can only receive a kidney from an $\mathrm{O}$ donor, type $\mathrm{A}$ patients can receive $\mathrm{A}$ or $\mathrm{O}$ kidneys, $\mathrm{B}$ patients $\mathrm{B}$ or $\mathrm{O}$ kidneys, and $\mathrm{AB}$ patients can receive a kidney from a donor of any blood type. Note that a patient with blood type $\mathrm{O}$ has the most difficulty finding a compatible donor, while $\mathrm{O}$ donors will never have a blood type incompatibility with any patient.

Tissue-type incompatibility ("positive crossmatch") is much less structured and has to do with the patient having preformed antibodies against one of the donor's proteins. Antibodies can arise from exposure to foreign proteins, e.g. through prior transplants, blood transfusions, or even childbirth. (Consequently mothers are less likely to be compatible with a kidney from the father of their children than from a random donor from the same population.)

An example helps illustrate why 3-way exchange is important. Consider a population of 9 incompatible patient-donor pairs. (A pair is denoted as type $\mathrm{x}-\mathrm{y}$ if the patient and donor are ABO blood-types $\mathrm{x}$ and y respectively.)

- Example: There are five pairs of patients who are blood-type incompatible with their donors, of types O-A, O-B, A-B, A-B, and B-A; and four pairs who are incompatible because of positive crossmatch, of types A-A, A-A, A-A and B-O. For simplicity in this example there are no positive crossmatches between patients and other patients' donors.

Then 6 transplants can result from the three possible 2-way exchanges, namely (A-B,B-A); (A-A,A-A); (B-O,O-B), where e.g. (A-B,B-A) denotes an exchange in which a pair of type (A-B) donates a B kidney to the patient in the pair of type (B-A) and receives an A kidney from the donor in that pair. When only 2-way exchanges are feasible, these three exchanges constitute a maximal set of exchanges: no other way of arranging exchanges would result in more than 6 transplants. But if 3-way exchanges are also feasible, then 8 transplants can be arranged via one 2-way exchange and two 3-way exchanges: (A-B,B-A); (A-A,A-A,A-A); (B$\mathrm{O}, \mathrm{O}-\mathrm{A}, \mathrm{A}-\mathrm{B}$ ). (In the 3 -way exchanges, the donor in the first pair donates to the patient in the 
second pair, the second donor donates to the third patient, and the third donor to the first patient. E.g. in the third exchange, the $\mathrm{O}$ donor from the $\mathrm{B}-\mathrm{O}$ pair donates to the $\mathrm{O}$ patient of the O-A pair, etc.)

The 3-way exchanges allow

1. an odd number of A-A pairs to be transplanted (instead of only an even number with 2 -way exchanges), and

2. an $\mathrm{O}$ donor to facilitate three transplants rather than only two.

While the difference between even and odd numbers of pairs will become proportionately smaller as the population of incompatible pairs grows, the importance of making good use of $\mathrm{O}$ donors will remain. $\mathrm{O}$ donors will be relatively rare among incompatible patient-donor pairs, because $\mathrm{O}$ donors are only incompatible with their intended recipient if there is a positive crossmatch.

In this example the only positive crossmatches were between patients and their own donors, but in actual populations there will be positive crossmatches between patients and other donors, particularly since a patient with a positive crossmatch with his own donor is more likely than average to be a highly sensitized patient who has antibodies to proteins in a high percentage of potential donors. In such a case there may be more configurations in which a 3 -way exchange will be helpful. For instance, suppose in our example the 2-way exchange (A-B, B-A) had been infeasible because of a positive crossmatch between the A patient in the first pair and the A donor in the second pair. A 3-way exchange (A-B, B-A, A-A) might nevertheless be possible, using one of the A-A pairs. However the case in which there are only blood-type incompatibilities (and no positive crossmatches) between patients and donors other than their own will allow us to establish an upper bound on the number of exchanges that are possible, since the presence of positive crossmatches can only reduce the set of feasible exchanges.

\section{The Model}

There are a number of kidney patients, each with an incompatible living donor. The incompatibility can be either a blood-type incompatibility or a tissue-type incompatibility (a positive crossmatch).

A two-way kidney exchange involves two patients each of whom is incompatible with her own donor but compatible with the other donor. When the two-way exchange is carried out, both patients receive a kidney from other's donor. A three-way kidney exchange involves three patients $\mathrm{i}, \mathrm{j}$, $\mathrm{k}$ each of whom is incompatible with her own donor but such that patient $\mathrm{i}$ is 
compatible with the donor of patient $\mathrm{j}$, patient $\mathrm{j}$ is compatible with the donor of patient $\mathrm{k}$, and patient $\mathrm{k}$ is compatible with the donor of patient $\mathrm{i}$. As in the case of two-way exchange, each patient involved in the trade receives a compatible kidney as a result of the three-way kidney exchange. A four-way kidney exchange is defined similarly.

While patients can have tissue-type incompatibility with their own donors, to establish an upper bound on the number of possible exchanges we will assume that:

Assumption 1 (Upper bound assumption). No patient is tissue-type incompatible with another patient's donor. ${ }^{5}$

We will derive analytical expressions for the maximum number of patients who can benefit from a feasible set of kidney exchanges among a large population of incompatible pairs, when the only constraints on exchange are imposed by

1. blood-type incompatibility, and

2. the number of patients allowed in each exchange.

Given the "upper bound" assumption, whether a patient can be part of a given exchange depends on her own blood-type together with her donor's blood-type. When we speak of a type $\mathrm{A}$-AB patient-donor pair, we mean a patient-donor pair in which the patient is of blood-type A while her incompatible donor is of blood-type AB. The number of type A-AB patient-donor pairs will be denoted by \#(A-AB). We use analogous notation for all 16 types of patient-donor pairs. We refer to type A-B pairs and type B-A pairs as opposite types, and use the same terminology for other types as well.

Patients with blood type $\mathrm{O}$ will be incompatible with their donors unless the donor also has blood type $\mathrm{O}$, and donors with blood type $\mathrm{AB}$ will be incompatible with their intended recipient unless she also is $\mathrm{AB}$. So pairs of these types will be over-represented in populations of incompatible patient-donor pairs. And, when exchanges are carried out, there will be higher demand for $\mathrm{O}$ kidneys than $\mathrm{A}$ kidneys and higher demand for A kidneys than AB kidneys. Similarly there will be higher demand for O kidneys than B kidneys and higher demand for B kidneys than AB kidneys. This puts pairs of types O-A, O-B, O-AB, A-AB, and B-AB at a disadvantage since they need a kidney that is in higher demand than the kidney they offer. So these type pairs will both occur more frequently and wait longer for an exchange than other

\footnotetext{
${ }^{5}$ When we later dispense with this assumption to consider populations with the tissue type incompatibilities found in the national patient population, we will see that, in large markets, this assumption isn't very consequential. The reason is that, although a patient who is tissue type incompatible with her own donor will likely have positive crossmatches with some other patients' donors also, in a sufficiently large population there will also be many donors with whom she has no tissue type incompatibility.
} 
types. Therefore the following is a natural assumption on any sufficiently large population of incompatible patient-donor pairs. ${ }^{6}$

Assumption 2. (Large Population of Incompatible Patient-Donor Pairs) Regardless of the maximum number of pairs allowed in each exchange, pairs of types O-A, O-B, O-AB, $\mathrm{A}-\mathrm{AB}$, and $\mathrm{B}-\mathrm{AB}$ are on the "long side" of the exchange in the sense that at least one pair of each type remains unmatched in each feasible set of exchanges. ${ }^{7}$

\subsection{Maximal-Size Two-Way Exchange}

For any non-negative number $k$, let $[k]$ denote the integer part of $k$, ie. the greatest integer no larger than $\mathrm{k}$.

Proposition 1. For any patient population obeying Assumptions 1 and 2, the maximum number of patients who can be matched with only two-way exchanges is:

$$
\begin{aligned}
& 2(\#(\mathrm{~A}-\mathrm{O})+\#(\mathrm{~B}-\mathrm{O})+\#(\mathrm{AB}-\mathrm{O})+\#(\mathrm{AB}-\mathrm{A})+\#(\mathrm{AB}-\mathrm{B})) \\
& \quad+(\#(\mathrm{~A}-\mathrm{B})+\#(\mathrm{~B}-\mathrm{A})-|\#(\mathrm{~A}-\mathrm{B})-\#(\mathrm{~B}-\mathrm{A})|) \\
& \quad+2\left(\left[\frac{\#(\mathrm{~A}-\mathrm{A})}{2}\right]+\left[\frac{\#(\mathrm{~B}-\mathrm{B})}{2}\right]+\left[\frac{\#(\mathrm{O}-\mathrm{O})}{2}\right]+\left[\frac{\#(\mathrm{AB}-\mathrm{AB})}{2}\right]\right)
\end{aligned}
$$

Proof: Each pair of type A-O is compatible with each pair of type O-A by Assumption 1, and types O-A are on the long side of the exchange by Assumption 2. Therefore each pair of type A-O (demanding a kidney that is less sought after than the one they provide) makes a two-way exchange possible. Moreover since only two-way exchanges are allowed, matching the A-O pair with a pair of a type other than O-A does not increase the size of the maximal set of exchanges. The same argument follows for each pair of types B-O, AB-O, AB-A, AB-B as well, explaining the term

$$
2(\#(\mathrm{~A}-\mathrm{O})+\#(\mathrm{~B}-\mathrm{O})+\#(\mathrm{AB}-\mathrm{O})+\#(\mathrm{AB}-\mathrm{A})+\#(\mathrm{AB}-\mathrm{B}))
$$

Now consider any maximal size match in which every pair of types A-O, B-O, AB-O, AB-A, $\mathrm{AB}-\mathrm{B}$ is matched with a pair of the opposite type. In the absence of any remaining types A-O,

\footnotetext{
${ }^{6}$ In particular, this assumption is met on each of the real and simulated populations of incompatible patientdonor pairs that we have examined that contain at least 25 pairs.

${ }^{7}$ It is worth emphasizing that this assumption will characterize large populations of incompatible patientdonor pairs, not patient-donor pairs in general. When we look at the whole population of patient-donor pairs (including the compatible pairs) there will in general be as many $\mathrm{O}$ donors as $\mathrm{O}$ patients. Note also that, in populations in which Assumption 2 is met, there will always be difficult choices to make about which $\mathrm{O}$ patients should receive the scarce O kidneys, even when attention is confined to maximal sets of exchanges. In game-theoretic terms, the strong core (the core defined by weak domination) of this market in which many kidneys are equally desirable, is empty (cf. Shapley and Scarf, 1974, Roth and Postlewaite, 1977, Quint and Wako, 2004).
} 
B-O, AB-O, AB-A, AB-B, pairs of types A-B and B-A can only be matched with a pair of the opposite type. Therefore, unless the two types have the same number of pairs, the longer side will have some pairs that remain unmatched. Since each pair on the shorter side can be matched with a pair on the longer side, $|\#(A-B)-\#(B-A)|$ pairs of types $A-B$, B-A remain unmatched under this maximal size set of exchanges, explaining the second term

$$
(\#(\mathrm{~A}-\mathrm{B})+\#(\mathrm{~B}-\mathrm{A})-|\#(\mathrm{~A}-\mathrm{B})-\#(\mathrm{~B}-\mathrm{A})|) \text {. }
$$

Finally when pairs of types A-O, B-O, AB-O, AB-A, AB-B are each matched with a pair of the opposite type, each pair of types A-A, B-B, AB-AB, O-O can only be matched with a pair of the same type. Therefore since only 2 -way exchanges are allowed, all pairs of type A-A can be matched whenever \#(A-A) is even and all but one can be matched whenever \#(A-A) is odd. The same argument follows for pairs of types B-B, AB-AB, O-O as well, explaining the last term

$$
2\left(\left[\frac{\#(\mathrm{~A}-\mathrm{A})}{2}\right]+\left[\frac{\#(\mathrm{~B}-\mathrm{B})}{2}\right]+\left[\frac{\#(\mathrm{O}-\mathrm{O})}{2}\right]+\left[\frac{\#(\mathrm{AB}-\mathrm{AB})}{2}\right]\right) .
$$

\subsection{Maximal-Size Two-Way and Three-Way Exchange}

The logistics of kidney exchange get harder as the number of patients in each exchange increases. Therefore one important question is how much of the gains from kidney exchange can be captured through two-way exchanges, and what are the marginal efficiency gains of three-way, four-way, and larger exchanges.

We will denote a three-way exchange as an ordered list of three patient-donor pairs, in which the donor of the first pair donates to the second patient, the donor of the second pair donates to the third patient, and the donor of the third pair donates to the first patient. We use similar terminology for four-way and larger exchanges.

While two-way kidney exchanges can capture most of the gains from exchange, they cannot capture all potential gains; at least some three-way exchanges are needed. There are several reasons for this:

1. Recall that when only two-way exchanges are allowed, one pair of type A-A remains unmatched if \#(A-A) is odd. There is a similar potential efficiency loss for each of the types B-B, AB-AB, and O-O. For almost all patient populations, that efficiency loss can be avoided once three-way exchanges are allowed. For example, unless there is only one type A-A pair, all type A-A pairs can be matched with each other through one three-way exchange and two-way exchanges for the others. Even when there is only one type A-A pair, she can be "appended" to a two-way exchange between an A-B pair and a B-A pair to form a three-way (B-A, A-A, A-B) exchange. 
PAIR 1

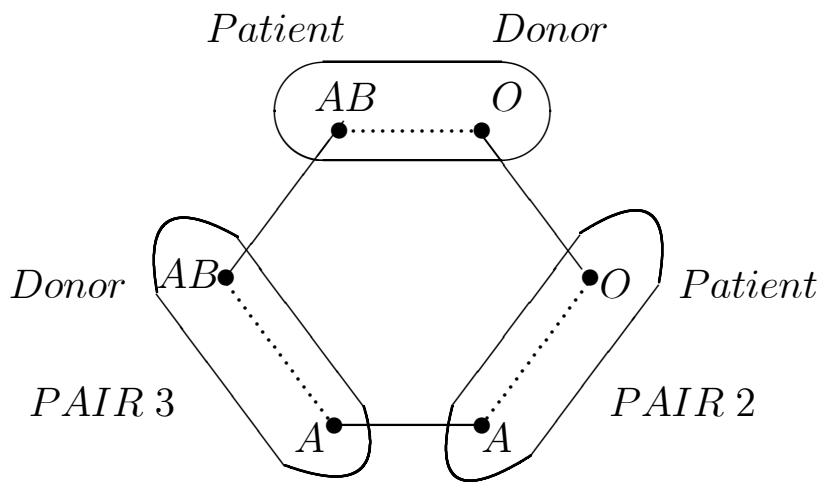

Patient
Donor
$P A I R 1$

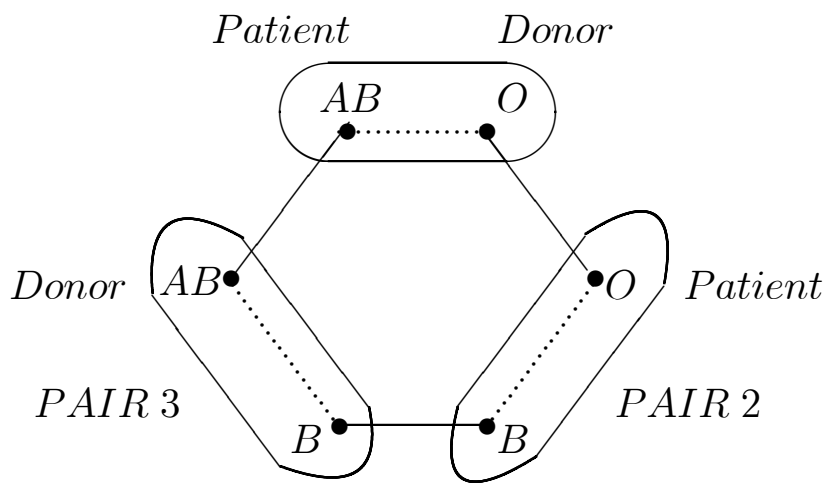

Patient
Donor

Figure 1: When three-way exchanges are feasible, each type AB-O pair can form a three-way exchange with two pairs on the long side.

2. With only two-way exchanges, under a maximal size match each type AB-O pair (with a rare $\mathrm{O}$ donor) is matched with a pair of one of the types $\mathrm{O}-\mathrm{AB}, \mathrm{O}-\mathrm{A}$, or O-B each of which is on the long side by Assumption 2. If three-way exchanges are allowed, each type $\mathrm{AB}-\mathrm{O}$ pair can trade with not one but two pairs on the long side through either a (AB-O, $\mathrm{O}-\mathrm{A}, \mathrm{A}-\mathrm{AB})$ exchange or $(\mathrm{AB}-\mathrm{O}, \mathrm{O}-\mathrm{B}, \mathrm{B}-\mathrm{AB})$ exchange. That increases the size of the maximal-size matching by one for each type AB-O pair (See Figure 1).

3. With only two-way exchanges, pairs of types A-B and B-A can be matched with each other, but $|\#(\mathrm{~A}-\mathrm{B})-\#(\mathrm{~B}-\mathrm{A})|$ pairs on the longer side remain unmatched. With threeway exchanges available, part or all of these $|\#(A-B)-\#(B-A)|$ pairs on the long side can also be matched, increasing the size of the maximal-size set of exchanges.

Terasaki, Gjertson and Cecka (1998) report that the frequency of types A-B and B-A are 0.05 and 0.03 respectively. So w.l.o.g. assume that:

Assumption 3. \#(A-B) > \#(B-A).

Consider a type B-O pair. In the absence of three-way exchanges, each such pair can be matched with one pair on the long side (such as a pair of the opposite type). However when three-way exchanges are available, a type B-O pair can form a three-way (B-O, O-A, 
PAIR 1

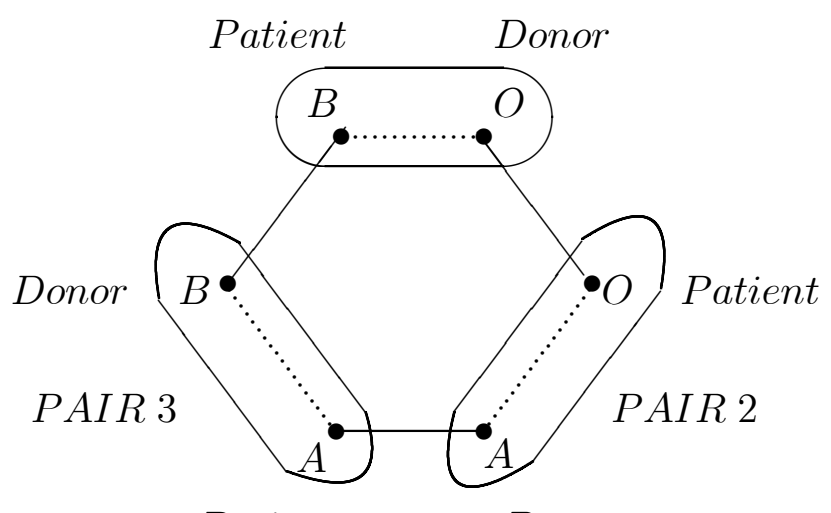

Patient
Donor
PAIR 1

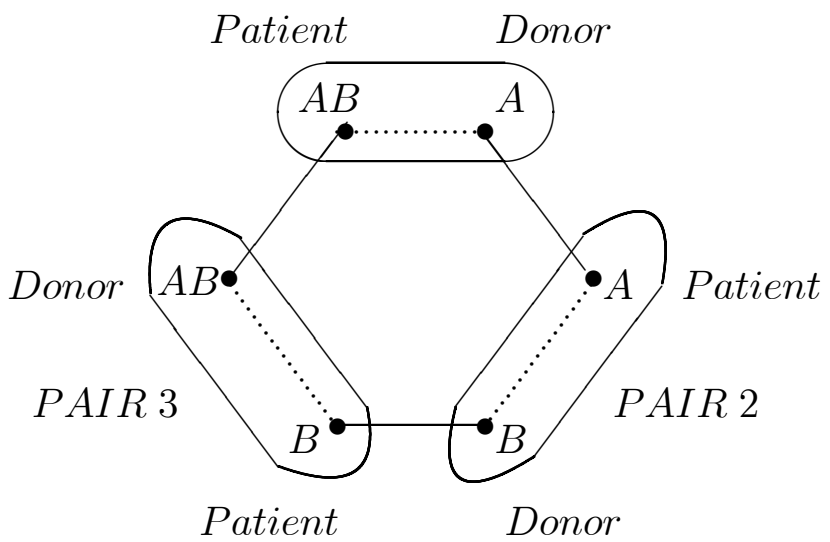

Figure 2: When three-way exchanges are feasible and \#(A-B) > \#(B-A), each type B-O pair can form a three-way exchange with two pairs on the long side. The same is also true for each type AB-A pair.

A-B) exchange together with two pairs each of which is on the long-side by Assumptions 2 and 3. Similarly while a type AB-A pair can be matched with one pair on the long side when only two-way exchanges are available, it can form a three way (AB-A, A-B, B-AB) exchange together with two pairs each of which is on the long-side by Assumptions 2 and 3 (See Figure 2). ${ }^{8}$

So of $|\#(\mathrm{~A}-\mathrm{B})-\#(\mathrm{~B}-\mathrm{A})|$ type A-B pairs who remain unmatched under two-way exchanges, as many as $(\#(B-O)+\#(A B-A))$ can be matched through three-way exchanges. Therefore such three-way exchanges increases the size of the maximal-size matching by

$$
\min \{(\#(\mathrm{~A}-\mathrm{B})-\#(\mathrm{~B}-\mathrm{A})),(\#(\mathrm{~B}-\mathrm{O})+\#(\mathrm{AB}-\mathrm{A}))\}
$$

To simplify the formula for the size of the maximal set of exchanges, we assume that none of the types A-A, B-B, AB-AB and O-O have only one pair. As we have already argued, when only one pair is present for any of these types it can be appended to a two-way exchange, but that considerably complicates the description of the size of the maximal-size set of exchanges.

\footnotetext{
${ }^{8}$ In case \#(B-A) > \#(A-B) instead, type A-O pairs and AB-B pairs become critical and each such pair can form a three-way exchange with two pairs on the long side.
} 
Assumption 4. There is either no type A-A pair or there are at least two of them. The same is also true for each of the types B-B, AB-AB, and O-O.

We are ready to summarize these observations in a proposition.

Proposition 2. For any patient population for which Assumptions 1-4 hold, the maximum number of patients who can be matched with two-way and three-way exchanges is:

$$
\begin{aligned}
2(\#) & (\mathrm{A}-\mathrm{O})+\#(\mathrm{~B}-\mathrm{O})+\#(\mathrm{AB}-\mathrm{O})+\#(\mathrm{AB}-\mathrm{A})+\#(\mathrm{AB}-\mathrm{B})) \\
& +(\#(\mathrm{~A}-\mathrm{B})+\#(\mathrm{~B}-\mathrm{A})-|\#(\mathrm{~A}-\mathrm{B})-\#(\mathrm{~B}-\mathrm{A})|) \\
& +(\#(\mathrm{~A}-\mathrm{A})+\#(\mathrm{~B}-\mathrm{B})+\#(\mathrm{O}-\mathrm{O})+\#(\mathrm{AB}-\mathrm{AB})) \\
& +\#(\mathrm{AB}-\mathrm{O})+\min \{(\#(\mathrm{~A}-\mathrm{B})-\#(\mathrm{~B}-\mathrm{A})),(\#(\mathrm{~B}-\mathrm{O})+\#(\mathrm{AB}-\mathrm{A}))\}
\end{aligned}
$$

To summarize, the marginal effect of three-way kidney exchanges is:

$$
\begin{aligned}
& \#(\mathrm{~A}-\mathrm{A})+\#(\mathrm{~B}-\mathrm{B})+\#(\mathrm{O}-\mathrm{O})+\#(\mathrm{AB}-\mathrm{AB})-2\left(\left[\frac{\#(\mathrm{~A}-\mathrm{A})}{2}\right]+\left[\frac{\#(\mathrm{~B}-\mathrm{B})}{2}\right]+\left[\frac{\#(\mathrm{O}-\mathrm{O})}{2}\right]+\left[\frac{\#(\mathrm{AB}-\mathrm{AB})}{2}\right]\right) \\
& +\#(\mathrm{AB}-\mathrm{O})+\min \{(\#(\mathrm{~A}-\mathrm{B})-\#(\mathrm{~B}-\mathrm{A})),(\#(\mathrm{~B}-\mathrm{O})+\#(\mathrm{AB}-\mathrm{A}))\}
\end{aligned}
$$

For large patient populations, the second line, that is the aggregate frequency of types AB$\mathrm{O}$, B-O and AB-A, account for essentially all gains from three-way exchanges (through the exchanges diagrammed in Figures 1 and 2). In small populations the first term, that is one additional patient matched for each of the types A-A, B-B, AB-AB and $\mathrm{O}-\mathrm{O}$ with an odd size, might account for a substantial part of the gains.

\subsection{Maximal-Size Two-Way, Three-Way and Four-Way Exchange}

We have already shown that each type AB-O can form a three-way exchange with two patients on the long side. In case four-way exchanges are allowed and if three-way exchanges cannot handle the entire difference between types A-B and B-A, a type AB-O patient can be matched with three patients on the long side to form a four-way exchange. So if $\#(A-B)>\#(B-A)$ as we assumed in Assumption 3, a type AB-O patient can form a four-way (AB-O, O-A, A-B, $\mathrm{B}-\mathrm{AB})$ exchange with three patients on the long side increasing the size of the maximal-size match by one (See Figure 3).

Proposition 3. For any patient population in which Assumptions 1-4 hold, the maximum number of patients who can be matched with two-way, three-way and four-way exchanges is:

$$
\begin{gathered}
2(\#(\mathrm{~A}-\mathrm{O})+\#(\mathrm{~B}-\mathrm{O})+\#(\mathrm{AB}-\mathrm{O})+\#(\mathrm{AB}-\mathrm{A})+\#(\mathrm{AB}-\mathrm{B})) \\
+(\#(\mathrm{~A}-\mathrm{B})+\#(\mathrm{~B}-\mathrm{A})-|\#(\mathrm{~A}-\mathrm{B})-\#(\mathrm{~B}-\mathrm{A})|)
\end{gathered}
$$




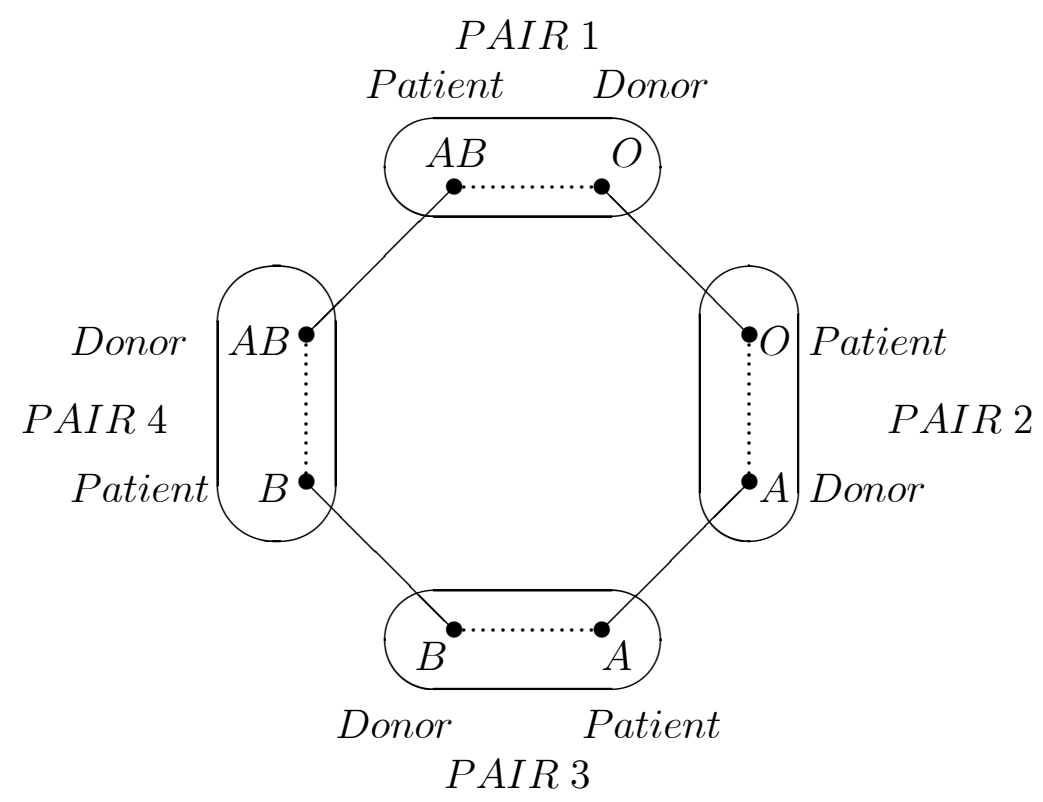

Figure 3: When four-way exchanges are feasible, each type AB-O pair can form a four-way exchange with three pairs on the long side.

$$
\begin{aligned}
& +(\#(\mathrm{~A}-\mathrm{A})+\#(\mathrm{~B}-\mathrm{B})+\#(\mathrm{O}-\mathrm{O})+\#(\mathrm{AB}-\mathrm{AB})) \\
& +\#(\mathrm{AB}-\mathrm{O})+\min \{(\#(\mathrm{~A}-\mathrm{B})-\#(\mathrm{~B}-\mathrm{A})),(\#(\mathrm{~B}-\mathrm{O})+\#(\mathrm{AB}-\mathrm{A})+\#(\mathrm{AB}-\mathrm{O}))\}
\end{aligned}
$$

Therefore in the absence of tissue-type incompatibilities between patients and other patients' donors, the marginal effect of four-way kidney exchanges is bounded above by the rate of the very rare AB-O type.

\subsection{Sufficiency of Two-Way, Three-Way and Four-Way Exchange}

So far we have derived analytical expressions for the maximum number of patients who can benefit from kidney exchange when the number of patients allowed in each exchange is no more than two, three, and four. We will next show that given Assumptions 1, 2, and 4, the number of patients who can benefit from exchange does not further increase when five-way or larger exchanges are feasible. We need a little additional notation to present this result.

Until now we described an exchange through the blood-types of the patients and their incompatible donors. In this section it will be useful to describe an exchange through the identities of the patients and their donors. For example when we speak of a three-way exchange

$$
E=\left(P_{1}-D_{1}, P_{2}-D_{2}, P_{3}-D_{3}\right)
$$


that means donor $D_{1}$ donates a kidney to patient $P_{2}$, donor $D_{2}$ donates a kidney to patient $P_{3}$, and donor $D_{3}$ donates a kidney to patient $P_{1}$. We will refer to any size exchange in a similar way.

An exchange is feasible if each donor in an exchange is compatible (both blood-type compatible and tissue-type compatible) with the patient to whom she donates. A matching is a collection of feasible exchanges such that no pair is part of more than one exchange. A maximal matching is one that includes as many patient-donor pairs as is feasible, i.e. it is a maximal-size matching.

Our next result indicates that, in the simple environments in which Assumptions 1, 2, and 4 apply, exchanges involving more than four pairs are never needed to achieve an efficient outcome.

Theorem (4-way exchange suffices): Consider a patient population for which Assumptions 1, 2, 4 hold and let $\mu$ be any maximal matching (when there is no restriction on the size of the exchanges that can be included in a matching). Then there exists a maximal matching $\nu$ which consists only of two-way, three-way, and four-way exchanges, under which the same set of patients benefit from exchange as in matching $\mu$.

Proof: Consider a patient population and a maximal matching $\mu$ as in the statement of the theorem. If $\mu$ is made of four-way or smaller exchanges then we are done. Otherwise we will construct a matching $\nu$ which is made of four-way or smaller exchanges and matches the same set of patients as matching $\mu$.

To simplify the exposition we will prove the theorem for the case in which the largest exchange in matching $\mu$ is five-way. In general the same proof can be used to show that given any maximal matching in which the largest exchange is of size $k>4$, there exists another matching which matches the same set of patients through $(k-1)$-way or smaller exchanges; and repeated application of this argument implies the desired result.

Let

$$
E=\left(P_{1}-D_{1}, P_{2}-D_{2}, P_{3}-D_{3}, P_{4}-D_{4}, P_{5}-D_{5}\right)
$$

be any five-way exchange in $\mu$. We will complete the proof by showing that all patient-donor pairs in this exchange can be matched via smaller exchanges without changing the set of patients who benefit from exchange.

Since there are four blood-types, there are at least two patients in exchange $E$ who have the same blood-type. Pick any two such patients. We have two cases to consider:

Case 1. Neither of the these patients receive a kidney from the incompatible donor of the other under exchange $E$. 
W.l.o.g. suppose these patients are $P_{1}$ and $P_{3}$. Under exchange $E$ patient $P_{1}$ receives a kidney from donor $D_{5}$ and patient $P_{3}$ receives a kidney from donor $D_{2}$. Since these two patients are of the same blood-type, by Assumption 1 donors $D_{2}$ and $D_{5}$ are compatible with both patients and hence the following two exchanges are feasible "dividing" exchange $E$ into two smaller exchanges:

$$
E^{\prime}=\left(P_{1}-D_{1}, P_{2}-D_{2}\right), \quad E^{\prime \prime}=\left(P_{3}-D_{3}, P_{4}-D_{4}, P_{5}-D_{5}\right)
$$

Case 2. One of these two patients receive a kidney from the incompatible donor of the other under exchange $E$.

W.l.o.g. suppose these patients are $P_{1}$ and $P_{2}$. Since $P_{1}$ receives a kidney from $D_{5}$, by Assumption 1 patient $P_{2}$ is also compatible with donor $D_{5}$ and hence the four-way exchange

$$
E^{*}=\left(P_{2}-D_{2}, P_{3}-D_{3}, P_{4}-D_{4}, P_{5}-D_{5}\right)
$$

is feasible. We will complete the proof by showing that the remaining pair $\left(P_{1}-D_{1}\right)$ can be included in an exchange without having an adverse impact on any patient who receives a kidney in the matching $\mu$.

Observe that while patients $P_{1}$ and $P_{2}$ are of the same blood-type, $P_{2}$ is compatible with donor $D_{1}$ and yet patient $P_{1}$ is incompatible. Therefore while $P_{1}$ is blood-type compatible with her donor $D_{1}$, she is tissue-type incompatible. We have two cases to consider:

Case 2a. Pair $\left(P_{1}-D_{1}\right)$ is one of the types A-O, B-O, AB-O, AB-A, or AB-B.

In this case pair $\left(P_{1}-D_{1}\right)$ is on the short side of the exchange and by Assumption 2 there exists a pair of the opposite type that is unmatched. By Assumption 1 pair $\left(P_{1}-D_{1}\right)$ can form a two-way exchange with this pair, increasing the total number of patients benefitting from exchange and contradicting that $\mu$ is of maximal-size. Hence this case cannot hold.

Case 2b. Pair $\left(P_{1}-D_{1}\right)$ is one of the types A-A, B-B, AB-AB, or O-O.

By Assumption 4 there is at least one other pair $\left(P_{6}-D_{6}\right)$ with the same type (and thus patients $P_{1}, P_{6}$ and donors $D_{1}, D_{6}$ all have the same blood-type). This pair is part of an exchange $\hat{E}$ under $\mu$ for otherwise pair $\left(P_{1}-D_{1}\right)$ can form a two-way exchange with them by Assumption 1 and this would contradict the maximality of matching $\mu$. If $\hat{E}$ is a two-way or three-way exchange then by Assumption 1 pair $\left(P_{1}-D_{1}\right)$ can be "appended" to this exchange (right before or right after pair $\left(P_{6}-D_{6}\right)$ ). If, on the other hand, $\hat{E}$ is a larger exchange, then pair $\left(P_{6}-D_{6}\right)$ can be removed from $\hat{E}$ to form a two-way exchange with pair $\left(P_{1}-D_{1}\right)$ which is feasible by Assumption 1 . Moreover since $P_{6}$ and $D_{6}$ are of the same blood type, the donor who donates a kidney to patient $P_{6}$ under $\hat{E}$ can instead donate a kidney to the patient who receives a kidney from $D_{6}$ under $\hat{E}$ and hence the remaining pairs in $\hat{E}$ can form an exchange with one less pair. 
Looking at the role that the Assumptions 1, 2, and 4 play in the theorem, we can restate it as follows. In a sufficiently large population of incompatible patient donor pairs, the only reasons that efficient exchange may require exchanges involving more than four pairs are idiosyncratic tissue type incompatibilities, and the presence of singleton patient-donor pairs with the same blood type. We next consider how much deviation from the above results we should expect in patient populations having the incidence of tissue type incompatibilities we see in the national patient population.

\section{Simulations using national patient characteristics}

In this section we dispense with the simplifying assumptions made so far, and turn to simulated data reflecting national patient characteristics. Specifically, we now look at populations in which a patient may have tissue type incompatibilities with many donors. This will allow us to see how good are the approximations derived above under the assumption that exchange was limited only by blood type incompatibilities.

The simulations reported here basically follow those of Saidman et. al 2005, with the addition that, for each simulated population we not only compute the actual maximal number of exchanges, but we also compute the predicted (upper bound) number based on the formulas derived above. ${ }^{9}$ (These formulas depend on the details of the simulation, insofar as they depend on the number of pairs of each type present in each simulated population.) We will see that the formulas predict the actual number of exchanges surprisingly well. That is, the upper bounds on the maximal number of exchanges when exchange is limited only by blood type incompatibility are not far above the numbers of exchanges that can actually be realized. In addition, only a small number of exchanges involving more than four pairs are needed to achieve efficiency in the simulated data.

\subsection{Patient and Donor Characteristics}

We consider samples of non-blood related patient-donor pairs in order to avoid the complications due to the impact of genetics on immunological incompatibilities. The characteristics such as the blood-types of patients and donors, the PRA (Percent Reactive Antibody) distribution of the patients, donor relation of patients, and the gender of the patients are generated using the empirical distributions of the U.S. Organ Procurement and Transplantation Network (OPTN) and the Scientific Registry of Transplant Recipients (SRTR) data (see Table 1). We consider all ethnicities in the data.

\footnotetext{
${ }^{9}$ Unlike in Saidman et al. we also consider four-way exchange.
} 


\subsection{Tissue-Type Incompatibility}

Tissue-type incompatibility (a positive crossmatch) is independent of blood-type incompatibility and arises when a patient has preformed antibodies against a donor tissue-type.

Patients in the OPTN/SRTR database are divided into the following three groups based on the odds that they have a crossmatch with a random donor:

1. Low PRA patients: Patients who have a positive crossmatch with less than $10 \%$ of the population.

2. Medium PRA patients: Patients who have a positive crossmatch with $10-80 \%$ of the population.

3. High PRA patients: Patients who have a positive crossmatch with more than $80 \%$ of the population.

Frequencies of low, medium, and high PRA patients reported in the OPTN/SRTR database are given in Table 1. Since a more detailed PRA distribution is unavailable in the medical literature, we will simply assume that:

- each low PRA patient has a positive crossmatch probability of $5 \%$ with a random donor,

- each medium PRA patient has a positive crossmatch probability of $45 \%$ with a random donor, and

- each high PRA patient has a positive crossmatch probability of $90 \%$ with a random donor.

We have already indicated that when a patient is female and the potential donor is her husband, it is more likely that they have a positive crossmatch due to pregnancies. Zenios, Woodle, and Ross (2001) indicate that while positive crossmatch probability is $11.1 \%$ between random pairs, it is $33.3 \%$ between female patients and their donor husbands. Equivalently, female patients' negative crossmatch probability (i.e. the odds that there is no tissuetype incompatibility) with their husbands is approximately $75 \%$ of the negative crossmatch probability with a random donor. Therefore we accordingly adjust the positive crossmatch probability between a female patient and her donor husband using the formula

$$
\mathrm{PRA}^{*}=100-0.75(100-P R A)
$$

and assume that 
- each low PRA female patient has a positive crossmatch probability of $28.75 \%$ with her husband,

- each medium PRA female patient has a positive crossmatch probability of $58.75 \%$ with her husband, and

- each high PRA female patient has a positive crossmatch probability of $92.25 \%$ with her husband.

\subsection{Patient-Donor Population Construction}

In our simulations, we randomly simulate a series of patient-donor pairs using the population characteristics explained above. Whenever a pair is compatible (both blood-type compatible and tissue-type compatible), the donor can directly donate to the intended recipient and therefore we do not include them in our sample. Only when they are either blood-type or tissue-type incompatible do we keep them, until we reach a sample size of $n$ incompatible pairs. We use a Monte-Carlo simulation size of 500 random population constructions for three population sizes of 25,50 and 100 .

\subsection{Outline of the Simulations}

For each sample of $\mathrm{n}$ incompatible patient-donor pairs, we find the maximum number of patients who can benefit from an exchange when both blood-type and tissue-type incompatibilities are considered, and

a. only two-way exchanges are allowed,

b. two-way and three-way exchanges are allowed,

c. two-way, three-way, and four-way exchanges are allowed, and

d. any size exchange is allowed.

In our simulations, to find the maximal number of patients who can benefit from an exchange when only two-way exchanges are allowed, we use a version of Edmonds' (1965) algorithm (see Roth, Sönmez, and Ünver, 2004b), and to find the maximal number of patients who can benefit from an exchange when larger exchanges are allowed, we use various integer programming techniques.

We compare these numbers with those implied by the analytical expressions we developed in order to see whether these formulae can be seen as close approximations or whether they 
merely represent crude upper-bounds. Since many high PRA patients cannot be part of any exchange due to tissue-type incompatibilities, we report two sets of upper-bounds induced by the formulae we developed:

1. For each sample we use the formulae with the raw data, and

2. for each sample we restrict our attention to patients each of whom can participate in at least one feasible exchange.

That is, in Table 2, "Upper bound 1" for each maximal allowable size exchange is the average over all simulated populations of the realization of the formula developed above for that size exchange (i.e. Propositions 1, 2, and 3 for maximal exchange sizes 2, 3, or 4 pairs) with the population size of $n=25,50$, or 100 . However in a given sample of size $n=25$, for example, there may be some patients who have no compatible donor with whom they can complete a feasible exchange, because of tissue type incompatibilities. So, for each size $k$ exchange that we consider, we look at the population of $n^{\prime}(k)<n$ of patient-donor pairs who can participate in a k-way or smaller exchange, and "Upper bound 2" in Table 2 reports the average over all populations for the realizations of the formulas using this smaller population of incompatible patient-donor pairs. Clearly Upper bound 2 provides a more precise (i.e. lower) upper bound to the number of exchanges that can be found. The fact that the difference between the two upper bounds diminishes as the population size increases reflects that, in larger populations, even highly sensitized patients are likely to find a compatible donor.

\subsection{Discussion of the Simulation Results}

The simulation results (which include tissue type incompatibilities) are very similar to the theoretical upper bounds we develop for the case with only blood type incompatibilities. While two-way exchanges account for most of the potential gains from exchange $(74 \%, 78 \%, 82 \%$ for population sizes of 25, 50, and 100 pairs respectively), the number of patients who benefit from exchange significantly increases when three or more pair exchanges are allowed. Consistent with the theory, three-way exchanges account for a large share of the remaining potential gains (77\%, 87\%, 94\% for a population sizes of 25, 50 and 100 pairs respectively). The theory that we developed in the absence of crossmatches is still predictive when there are crossmatches: virtually all possible gains from trade are achieved with two-way, three-way and four-way exchanges, especially when the population size is large (See Table 2). ${ }^{10}$

\footnotetext{
${ }^{10}$ When the population size is 100 incompatible pairs, in 485 of the 500 simulated populations the maximum possible gains from trade are achieved when no more than four pairs are allowed to participate in an exchange.
} 


\section{Concluding remarks}

This paper has two themes. The first is intensely practical, and concerns the infrastructure that needs to be prepared to efficiently conduct kidney exchange among incompatible patient-donor pairs. The second is more general, and concerns the role of markets in facilitating exchange even in the absence of a medium of exchange.

On the practical side, we have shown why it will be important to develop the infrastructure to identify and to perform three-way as well as two-way kidney exchanges, since the efficient utilization of $\mathrm{O}$ donors often requires three-way exchanges. Although the identification of maximal two and three-way exchanges is a computationally hard problem (unlike the identification of maximal two-way exchanges; Roth, Sönmez, and Ünver, 2004b), it appears that instances of practical size can be readily solved with conventional integer programming software. And, while performing three-way exchanges requires six simultaneous surgeries, this will often be feasible, particularly when the patients are at different transplant centers. It seems likely that the most usual logistical arrangement will be for the donor to travel to the designated patient's transplant center. So, for example, in the case of a three way exchange in which each patient is at a different hospital, this means that each of three hospitals will only be performing two surgeries, which they would be required to perform in any live-donor kidney transplant. ${ }^{11}$ As Proposition 2 explains, and Table 2 demonstrates, the gains from including the possibility of three-way exchange are substantial, and substantially greater than the further marginal gains from four-way exchange (Proposition 3 and Table 2). Thus, at least initially we will be searching for two and three-way exchanges.

\subsection{M\&M's: Money and Markets}

More generally, this paper is about how markets facilitate exchange. Jevons (1876) famously suggests that the primary difficulties with barter arise from the absence of, and can be solved by the introduction of, a medium of exchange. We argue that many of the problems that Jevons identifies as resulting from an absence of money would continue to be problems (even with money) in the absence of a sufficiently thick market, and that many of the benefits Jevons sees as flowing from the presence of money result at least in part from the presence of a market. Kidney exchange allows us to see which of the market effects can still be achieved in the absence of money.

\footnotetext{
${ }^{11}$ The logistics of three-way exchanges will remain more difficult than two-way exchanges, even when three hospitals are involved. A three way exchange involves scheduling surgery for six patients, all of whom must be ready for surgery at the same time, and will consequently have higher probability of being delayed or cancelled due to changing health conditions than a two-way exchange that involves only four patients.
} 
In the absence of a thick market, only a very small number of kidney exchanges have so far been accomplished. We show that a thick market organized by a clearinghouse provides many of the benefits that Jevons attributed to the presence of a medium of exchange. If we confine our attention narrowly to a sufficiently large population of patient-donor pairs, all of the efficiencies of exchange can be achieved without money, primarily in exchanges involving no more than three pairs. Table 2 shows that about $60 \%$ of the incompatible pairs can benefit in this way, with those that cannot being the pairs on the long side of the market, primarily $\mathrm{O}$ blood type patients with $\mathrm{A}$ or $\mathrm{B}$ donors. To benefit these patients it would be necessary to increase the supply of $\mathrm{O}$ type donors.

Two thought experiments about how to do this help illustrate the parallel roles of money and markets. Suppose that instead of confining our attention to incompatible patient-donor pairs, we considered exchange among the entire population of patient-donor pairs, i.e. suppose we also included in the pool of potential exchangers those pairs in which the donor could give a kidney to the intended recipient directly. ${ }^{12}$ In that case there would no longer be a shortage of $\mathrm{O}$ donors, and simulations show that, in populations of 100 (compatible and incompatible) patient-donor pairs, it would be possible to arrange transplants for over $90 \%$ of the patientdonor pairs (Roth, Sönmez, and Ünver 2005). Of course, these benefits, achieved without the use of money, accrue only to those patients who have a willing kidney donor. In contrast, if the legal/ethical/social objections to a fully monetized market were to be resolved, and money could be exchanged to recruit donors from the general population, then it would presumably be possible to arrange transplants for a high percentage of all patients in need of one.

While we are on the subject of the parallel roles of money and markets, the structure of efficient exchange in the case of kidneys allows us to address, without the use of money, another of money's traditional roles.

"A second difficulty arises in barter. At what rate is any exchange to be made? If a certain quantity of beef be given for a certain quantity of corn, and in like manner corn be exchanged for cheese, and cheese for eggs, and eggs for flax, and so on, still the question will arise - How much beef for how much flax, or how much of any one commodity for a given quantity of another?" Jevons (1876, Chapter 1)

\footnotetext{
${ }^{12}$ At present in New England, only incompatible pairs may be registered for exchange. As we noted in Roth, Sönmez, and Ünver (2005): "It seems likely that until exchange becomes well established, only incompatible patient-donor pairs will be included, as surgeons will be reluctant to advise compatible pairs not to proceed with their own transplant. However, as exchange becomes more routine, there will be opportunities for mutually beneficial exchange between e.g. a 25-year-old patient with a compatible 50-year-old donor and a 50-year-old patient with an incompatible 25 -year-old donor."
} 
As we have seen, if the object is to facilitate as many transplants as possible among incompatible patient-donor pairs, then we can determine the rates of exchange. For example a (B-O) pair on the short side of the market can exchange with two pairs on the long side of the market (Figure 2), while an even rarer (AB-O) pair can exchange with three pairs on the long side (Figure 3$).{ }^{13}$

\subsection{Practical Market Design}

As economists are more frequently called upon to design markets, ${ }^{14}$ and not merely to study them at arms length, there will be new opportunities for understanding how markets work, and what obstacles they face in achieving efficient allocation.

Kidney exchange, in the context of the legal/social/ethical prohibitions on the buying and selling of organs, gives us a chance to look closely at the exchange of indivisible goods, without a medium of exchange, in an environment in which the structure of efficient exchange can be analyzed. One of the main contributions that a centralized clearinghouse can make in this context is to establish a sufficiently thick market so that double and triple coincidences of wants can be identified and consummated.

In general, clearinghouses seem to be in most demand in markets that have suffered failures with respect to the thickness of the market, or congestion in making and processing enough offers, or that have incentive problems that make it unsafe for participants to provide information needed for efficient allocation. For example, clearinghouses recently designed for other markets in which monetary transfers are considered inappropriate have involved the allocation of public school places in New York City (where the clearinghouse solved a congestion problem), and in Boston, where the existing allocation system had an incentive problem (see Abdulkadiroğlu and Sönmez 2003, Abdulkadiroğlu, Pathak, and Roth, 2005, and Abdulkadiroğlu, Pathak, Roth, and Sönmez, 2005). Clearinghouses are also used in markets in which monetary wages are entirely appropriate, such as the labor markets for new doctors (see e.g. Roth, 1984, Roth and Peranson, 1999), and for medical specialists of various sorts (see e.g. Niederle and Roth, 2005). And there has been recent attention to how decentralized markets deal well or badly with some of the same problems that clearinghouses can be used to solve (in e.g. the markets for college admissions and financial aid, law clerks, psychologists, gastroenterologists, collectables, etc., see e.g. Avery et al. 2001, 2003, Niederle and Roth, 2003, 2004, Ockenfels and Roth 2004,

\footnotetext{
${ }^{13}$ Note that if the objective is to maximize the total number of transplants, then patient-donor pairs of type $\mathrm{O}-\mathrm{AB}$ may be adversely affected, if the AB-O types are used in four way exchanges. Matching O-AB pairs will therefore often require reducing the total number of patients who can be matched.

${ }^{14}$ cf. e.g. Milgrom (2004), Roth (2002), Wilson (2002).
} 
Roth and Xing 1997).

All of these markets, like kidney exchange, are matching markets, in the sense that it matters who transacts with whom (in contrast to anonymous commodity markets). And the fact that some of these markets can do much of their work without money at all supports the view that many matching markets in which money is freely available nevertheless do not clear by price adjustments alone. (Departments of economics don't hire professors by announcing a price and seeing who comes...)

Viewed in this light, the present study is part of the growing investigation into how markets are and need to be designed to achieve efficiency, taking into account the particular obstacles that they encounter. This line of work, which is given focus by the demand for practical designs for particular markets, directs our attention to the many still poorly understood details of market clearing, in a way that can in the long term only deepen our understanding of how markets work in general.

\section{References}

[1] A. Abdulkadiroğlu, P. A. Pathak, and A. E. Roth (2005) "The New York City high school match," American Economic Review, Papers and Proceedings, May, in press.

[2] A. Abdulkadiroğlu, P. A. Pathak, A. E. Roth, and T. Sönmez (2005) "The Boston public school match," American Economic Review, Papers and Proceedings, May, in press.

[3] A. Abdulkadiroğlu and T. Sönmez (2003) "School choice: A mechanism design approach," American Economic Review 93: 729-747.

[4] M. Abecassis et al. (2002) "Consensus statement on the live organ donor," The Journal of the American Medical Association 284: 2919-2926.

[5] C. Avery, A. Fairbanks, and R. Zeckhauser (2003) The Early Admissions Game: Joining the Elite, Harvard University Press, Cambridge, MA.

[6] C. Avery, C. Jolls, R. A. Posner, and A. E. Roth (2001) "The market for federal judicial law clerks," University of Chicago Law Review 68: 793-902.

[7] G. S. Becker and J. J. Elias (2002) "Introducing incentives in the market for living organ donations," working paper, University of Chicago. 
[8] F. L. Delmonico (2004) "Exchanging kidneys - Advances in living-donor transplantation," The New England Journal of Medicine 350: 1812-1814.

[9] J. Edmonds (1965) "Paths, trees, and flowers," Canadian Journal of Mathematics 17: 449-467.

[10] J. W. Hatfield (2005) "Pairwise kidney exchange: Comment," working paper, Stanford University.

[11] W. S. Jevons (1876) Money and the Mechanism of Exchange. New York: D. Appleton and Co. [Online] available from http://www.econlib.org/library/YPDBooks/Jevons/jvnMME1.html; accessed 30 April 2005

[12] M. Lucan, P. Rotariu, D. Neculoiu, and G. Iacob (2003) "Kidney exchange program: A viable alternative in countries with low rate of cadaver harvesting," Transplantation Proceedings 35: 933-934.

[13] C. N. McKinney, M. Niederle, and A. E. Roth (2005) "The collapse of a medical labor clearinghouse (and why such failures are rare)," American Economic Review, in press.

[14] P. Milgrom (2004) Putting Auction Theory to Work, Cambridge University Press.

[15] M. S. Nadel and C. A. Nadel (2005) "Using reciprocity to motivate organ donations," Yale Journal of Health Policy, Law and Ethics 5: 293-325.

[16] M. Niederle and A. E. Roth (2003) "Unraveling reduces mobility in a labor market: Gastroenterology with and without a centralized match," Journal of Political Economy 111: $1342-1352$.

[17] M. Niederle and A. E. Roth (2004) "Market culture: How norms governing exploding offers affect market performance," working paper, Stanford University and Harvard University.

[18] M. Niederle and A. E. Roth (2005) "The gastroenterology fellowship market: Should there be a match?" American Economic Review, Papers and Proceedings, May, in press.

[19] A. Ockenfels and A. E. Roth (2004) "Convergence of prices for a new commodity: 'Iraq most wanted' cards on eBay," working paper, Köln University and Harvard University.

[20] T. Quint and J. Wako (2004) "On houseswapping, the strict core, segmentation, and linear programming," Mathematics of Operations Research 29: 861-877. 
[21] F. T. Rapaport (1986) "The case for a living emotionally related international kidney donor exchange registry," Transplantation Proceedings 18: 5-9.

[22] L. F. Ross, D. T. Rubin, M. Siegler, M. A. Josephson, J. R. Thistlethwaite, Jr., and E. S. Woodle (1997) "Ethics of a paired-kidney-exchange program," The New England Journal of Medicine 336: 1752-1755.

[23] L. F. Ross and E. S. Woodle (2000). "Ethical issues in increasing living kidney donations by expanding kidney paired exchange programs," Transplantation 69: 1539-1543.

[24] A. E. Roth (1984) "The evolution of the labor market for medical interns and residents: a case study in game theory," Journal of Political Economy 92: 991-1016.

[25] A. E. Roth (2002) "The economist as engineer: game theory, experimental economics and computation as tools of design economics," Econometrica 70: 1341-1378.

[26] A. E. Roth and E. Peranson (1999) "The redesign of the matching market for American physicians: some engineering aspects of economic design," American Economic Review 89: 748-780.

[27] A. E. Roth and A. Postlewaite (1977) "Weak versus strong domination in a market with indivisible goods," Journal of Mathematical Economics 4: 131-137.

[28] A. E. Roth, T. Sönmez, and M. U. Ünver (2004a) "Kidney exchange," Quarterly Journal of Economics 119: 457-488.

[29] A. E. Roth, T. Sönmez, and M. U. Ünver (2004b) "Pairwise kidney exchange," National Bureau of Economic Research, working paper no. w10698, August, Journal of Economic Theory in press.

[30] A. E. Roth, T. Sönmez, and M. U. Ünver (2005) "A kidney exchange clearinghouse in New England," American Economic Review, Papers and Proceedings, May, in press.

[31] A. E. Roth and X. Xing (1997) "Turnaround time and bottlenecks in market clearing: decentralized matching in the market for clinical psychologists," Journal of Political Economy 105: 284-329.

[32] S. L. Saidman, A. E. Roth, T. Sönmez, M. U. Ünver, and F. L. Delmonico (2005) "Increasing the opportunity of live kidney donation by matching for two and three way exchanges," working paper, Massachusetts General Hospital, Harvard University, Koç University, New England Organ Bank. 
[33] D. L. Segev, S. E. Gentry, D. S. Warren, B. Reeb, and R. A. Montgomery (2005) "Kidney paired donation and optimizing the use of live donor organs," The Journal of American Medical Association 293: 1883-1890.

[34] L. Shapley and H. Scarf (1974) "On cores and indivisibility," Journal of Mathematical Economics 1: 23-28.

[35] P. I. Terasaki, D. W. Gjertson, and J. M. Cecka (1998) "Paired kidney exchange is not a solution to ABO incompatibility," Transplantation 65: 291.

[36] R. B . Wilson (2002) "Architecture of power markets," Econometrica 70: 1299-1340.

[37] S. Zenios, E. S. Woodle, and L. F. Ross (2001) "Primum non nocere: avoiding harm to vulnerable wait list candidates in an indirect kidney exchange," Transplantation 72 : $648-54$. 
Table 1: Patient and living donor distributions used in simulations based on OPTN/SRTR Annual Report in 2003, for the period 1993-2002, retrieved from http://www.optn.org on 11/22/2004. Patient characteristics are obtained using the new waiting list registrations data, and living donor relatinal type distribution is obtained from living donor transplants data.

\begin{tabular}{|c|c|}
\hline A. Patient ABO Blood Type & Frequency \\
\hline $\mathrm{O}$ & $48.14 \%$ \\
\hline $\mathrm{A}$ & $33.73 \%$ \\
\hline $\mathrm{B}$ & $14.28 \%$ \\
\hline $\mathrm{AB}$ & $3.85 \%$ \\
\hline \hline B. Patient Gender & Frequency \\
\hline Female & $40.90 \%$ \\
\hline Male & $59.10 \%$ \\
\hline \hline C. Unrelated Living Donors & Frequency \\
\hline Spouse & $48.97 \%$ \\
\hline Other & $51.03 \%$ \\
\hline \hline E. PRA Distribution & Frequency \\
\hline Low PRA & $70.19 \%$ \\
\hline Medium PRA & $20.00 \%$ \\
\hline High PRA & $9.81 \%$ \\
\hline
\end{tabular}


Table 2: Simulation results about average number of patients actually matched and predicted by the formulae to be matched. The standard errors of the population are reported in parantheses. The standard errors of the averages are obtained by dividing population standard errors by square root of the simulation number, 22.36 .

\begin{tabular}{|c|c|c|c|c|c|}
\hline \multirow[b]{2}{*}{$\begin{array}{l}\text { Pop. } \\
\text { Size }\end{array}$} & \multirow[b]{2}{*}{ Method } & \multicolumn{4}{|c|}{ Type of Exchange } \\
\hline & & Two-way & $\begin{array}{l}\text { Two-way, } \\
\text { Three-way }\end{array}$ & $\begin{array}{c}\text { Two-way, } \\
\text { Three-way, } \\
\text { Four-way }\end{array}$ & $\begin{array}{c}\text { No } \\
\text { Constraint }\end{array}$ \\
\hline \multirow{3}{*}{$\mathrm{n}=\mathbf{2 5}$} & Simulation & $\begin{array}{c}8.86 \\
(3.4866)\end{array}$ & $\begin{array}{c}11.272 \\
(4.0003)\end{array}$ & $\begin{array}{c}11.824 \\
(3.9886)\end{array}$ & $\begin{array}{c}11.992 \\
(3.9536)\end{array}$ \\
\hline & Upper bound 1 & $\begin{array}{c}12.5 \\
(3.6847)\end{array}$ & $\begin{array}{c}14.634 \\
(3.9552)\end{array}$ & $\begin{array}{c}14.702 \\
(3.9896)\end{array}$ & \\
\hline & Upper bound 2 & $\begin{array}{c}9.812 \\
(3.8599) \\
\end{array}$ & $\begin{array}{c}12.66 \\
(4.3144)\end{array}$ & $\begin{array}{c}12.892 \\
(4.3417)\end{array}$ & \\
\hline \multirow{3}{*}{$\mathrm{n}=50$} & Simulation & $\begin{array}{c}21.792 \\
(5.0063)\end{array}$ & $\begin{array}{c}27.266 \\
(5.5133)\end{array}$ & $\begin{array}{c}27.986 \\
(5.4296)\end{array}$ & $\begin{array}{c}28.09 \\
(5.3658)\end{array}$ \\
\hline & Upper bound 1 & $\begin{array}{c}27.1 \\
(5.205)\end{array}$ & $\begin{array}{c}30.47 \\
(5.424)\end{array}$ & $\begin{array}{c}30.574 \\
(5.4073)\end{array}$ & \\
\hline & Upper bound 2 & $\begin{array}{c}23.932 \\
(5.5093)\end{array}$ & $\begin{array}{l}29.136 \\
(5.734)\end{array}$ & $\begin{array}{c}29.458 \\
(5.6724)\end{array}$ & \\
\hline \multirow{3}{*}{$n=100$} & Simulation & $\begin{array}{c}49.708 \\
(7.3353)\end{array}$ & $\begin{array}{l}59.714 \\
(7.432)\end{array}$ & $\begin{array}{c}60.354 \\
(7.3078)\end{array}$ & $\begin{array}{l}60.39 \\
(7.29)\end{array}$ \\
\hline & Upper bound 1 & $\begin{array}{c}56.816 \\
(7.2972) \\
\end{array}$ & $\begin{array}{c}62.048 \\
(7.3508) \\
\end{array}$ & $\begin{array}{c}62.194 \\
(7.3127) \\
\end{array}$ & \\
\hline & Upper bound 2 & $\begin{array}{c}53.496 \\
(7.6214)\end{array}$ & $\begin{array}{c}61.418 \\
(7.5523)\end{array}$ & $\begin{array}{c}61.648 \\
(7.4897)\end{array}$ & \\
\hline
\end{tabular}

